\title{
Anterior tibial compartment syndrome following femoral artery perfusion
}

\author{
B. V. PALMER and J. L. MER CER \\ Department of Thoracic Surgery, St. Bartholomew's Hospital, London
}

\begin{abstract}
Palmer, B. V., and Mercer, J. L. (1973). Thorax, 28, 492-494. Anterior tibial compartment syndrome following femoral artery perfusion. Four patients are described who developed the anterior tibial compartment syndrome following open heart surgery. It occurred in the limb used for femoral artery perfusion in each patient. In two it was known that the collateral circulation to the limb was also considerably impaired. It is suggested that prolonged limb ischaemia is the major initiating factor.

The treatment of these patients is discussed and although one patient was successfully treated conservatively, surgical treatment is advisable.
\end{abstract}

The acute anterior tibial compartment syndrome first described by Vogt (1943) most commonly occurs following lower limb exercise, usually of an intensive nature in a person not used to vigorous activity. The syndrome consists of a dull ache in the anterior part of the lower leg, becoming increasingly painful during or soon after exercise. There is marked tenderness and swelling of the anterior compartment muscles associated with their weakness or paralysis. The dorsalis pedis pulse may or may not be absent, and there may be damage to the deep peroneal nerve.

Other less common causes include an embolus in the anterior tibial artery or a fracture of the tibia.

This paper describes four patients who developed this syndrome following cardiopulmonary bypass in which the femoral artery had been cannulated.

\section{CASE REPORTS}

PATIENT 1 A 38-year-old man had one year's history of angina. On examination he had the signs of tight aortic stenosis. Transseptal catheterization showed a left ventricular pressure of $220 / 140 \mathrm{mmHg}$ with an aortic pressure of $90 / 55 \mathrm{mmHg}$. All pulses were normal.

On 9 April 1968 the aortic valve was replaced using left femoral artery perfusion. A heavily calcified bicuspid valve was replaced by a Starr-Edwards prosthesis. When he came off bypass the cardiac output fell rapidly and bypass was resumed. The patient was eventually taken off bypass after a total bypass time of 4 hours 24 minutes. His temperature during bypass was maintained at around $26^{\circ} \mathrm{C}$. The arterial can- nula occluded the circumflex iliac and profunda femoris as well as the femoral artery. It was in place for about 40 minutes after the patient came off bypass and after protamine had been given.

It was noted immediately after the operation that the left lower leg was swollen, tense, and warm. Theo ankle was not easily flexed. The posterior tibial and $\vec{\overrightarrow{ }}$ dorsalis pedis pulses were reduced in volume. Anterior tibial compartment syndrome was diagnosed. As the patient's condition was poor it was decided to treat the leg conservatively. He required isoprenaline infusion to maintain the blood pressure above 70 F $\mathrm{mmHg}$ and a lignocaine infusion to control ventricular 은 ectopic beats. Three days later he had a tracheostomy. $\frac{\mathbb{x}}{x}$ After a stormy postoperative period, which included $\dot{0}$ four episodes of asystole requiring resuscitation, he eventually recovered.

The left calf remained swollen with complete foot drop. There was little sensation below the knee and faradic tests showed that there was no contraction of $\stackrel{?}{\square}$ the anterior tibial group of muscles and diminished $D$ function of the calf muscles. The small muscles on the dorsum of the foot responded minimally to $N$ faradism and it was concluded that there was also? considerable nerve damage.

Four years later the foot drop is unchanged but N he is able to work normally without inconvenience or special appliances.

PATIENT 2 A 22-year-old woman had a heart murmur of aortic stenosis first noticed at the age of 2 years. $\stackrel{\mathcal{P}}{+}$ At 13 years she became increasingly tired on exertion and following investigation she had an open aortic valvotomy. This resulted in some aortic incompetence. $\overrightarrow{\mathbb{D}}$

In June 1968 she was readmitted with a view to $\stackrel{?}{\oplus}$ further surgery. During the previous year she had $\stackrel{\mathbb{Q}}{\mathscr{2}}$ noticed increasing tiredness, dyspnoea on exertion, and $\bar{O}$ intermittent peripheral cyanosis but no other evidence of heart failure. 
On examination she had the signs of marked aortic incompetence, a blood pressure of $140 / 30 \mathrm{mmHg}$, and left ventricular hypertrophy. Angiography confirmed gross aortic regurgitation and a dilated left ventricle.

On 25 June 1968 a homograft aortic valve replacement was performed using a right femoral artery cannula. The total bypass time was 3 hours 58 minutes during which her temperature was maintained between $24^{\circ} \mathrm{C}$ and $26^{\circ} \mathrm{C}$.

Soon after her return to the recovery ward it was noticed that the right anterior compartment was swollen and tender. The dorsalis pedis pulse was present but weak. The anterior tibial compartment syndrome was diagnosed. On the evening of operation she was returned to the operating theatre and a longitudinal incision was made over the anterior compartment. The deep fascia was incised and bulging oedematous muscle exposed. The fascia was loosely approximated with catgut, leaving the edges $2.5 \mathrm{~cm}$ apart. The skin layer was closed. Postoperatively she made a good recovery. Eleven days later there was some reduction in the strength of dorsiflexion of the right ankle but after six weeks this was almost back to normal.

PATIENT 3 A 19-year-old boy had had rheumatic fever at the age of 7 years. Subsequently he had intermittent palpitations but these did not limit his activities.

Over the previous three months he complained of fatigue, increasing dyspnoea, and palpitations on exercise, although he was able to walk one mile on the flat.

On examination he had the signs of marked aortic incompetence with a blood pressure of $150 / 30 \mathrm{mmHg}$.

On 17 October 1968 his aortic valve was replaced with a Starr-Edwards prosthesis. The left femoral artery was cannulated. Total bypass time was 2 hours 47 minutes during which period his temperature was maintained at between $26^{\circ} \mathrm{C}$ and $28^{\circ} \mathrm{C}$. Postoperatively he maintained a good blood pressure of about $115 / 80 \mathrm{mmHg}$ after operation and all his peripheral pulses were present. Twelve hours after operation it was noticed that the right posterior tibial compartment was swollen and tender, and two hours later the anterior compartment was becoming similarly affected. The dorsalis pedis was just palpable, but there was no posterior tibial pulse. It was decided that he had both anterior and posterior compartment syndromes and the patient was returned to the operating theatre. The right deep fascia was incised longitudinally over both the anterior and posterior compartments and oedematous muscle bulged out. The skin incisions were left open.

The skin incisions of the posterior compartments were closed on the seventeenth day and that of the anterior compartment on the twenty-third day. On discharge he was walking well with only minimal weakness of his anterior compartment muscles.

PATIENT 4 A 38-year-old man had three episodes of rheumatic fever when a child. Over the previous two years he had had some shortness of breath on exercise.
In March 1972 he began to develop anginal pain on exercise, which was relieved by glyceryl trinitrate.

On examination he was a well built man with signs of moderate aortic incompetence and mild mitral stenosis which were not considered severe enough to account for his angina. Coronary angiography confirmed severe stenosis of both coronary arteries.

On 10 October 1972 he underwent aortic valve replacement and right coronary bypass vein graft. The left femoral artery was exposed for cannulation, but when the artery was opened gross atheroma was found with only a small lumen posteriorly. Aortic cannulation was therefore performed. The femoral artery and profunda (which was damaged during mobilization) were occluded until they were repaired at the end of the operation. Total bypass time was 3 hours 2 minutes. On return to the ward there was some swelling and firmness of the left lower leg although the pulses were normal. There was slight reduction in the power of foot flexion below the knee; the patient complained of numbness and sensation was diminished. Anterior and posterior tibial compartment syndromes were diagnosed.

Over the following hours and days there was no worsening in the condition of the leg and conservative treatment was decided upon. At the end of three weeks the swelling had virtually disappeared. There was no weakness, though slight shortening of the calf muscles had occurred.

\section{DISCUSSION}

Considering the following features, the diagnosis of tibial compartment syndrome was preferred to deep vein thrombosis:

1. the swelling was noted within a few hours of operation in all cases ;

2. the anterior compartment was involved in all cases ;

3. there was prolonged swelling of the muscles resulting in their contracture and weakness ;

4. at operation in cases 2 and 3 the muscle was pale, swollen, and oedematous ;

5 . the arterial pulses were reduced in cases 1,2 , and 3 ;

6. evidence of associated nerve damage was seen in case 1.

The aetiology of the anterior compartment syndrome is still uncertain and it is probable that more than one mechanism is responsible. The generally accepted theory is that as a result of muscle damage oedema and swelling occur which account for the bulging out of the underlying muscle on incision of the overlying fascia (cases 2 and 3). The anterior compartment is an enclosed space with rigid walls, and the muscle swelling results in an increased pressure in the compartment. This will produce venous stasis and also arterial occlusion, resulting eventually in ischaemic necrosis of muscle and nerves. 
Carter, Richards, and Zachary (1949) have discussed the possible factors involved in producing the ischaemia. It is relevant to see how each of their suggestions could be involved in causing the syndrome in our patients.

The pathology of the condition is identical to Volkmann's ischaemia, and to muscle ischaemia following arterial wounds.

Our four patients had trauma to their femoral arteries. However, the fact that the posterior tibial pulse remained intact and that the disappearance of the dorsalis pedis tends to be a late feature is against any theory invoking arterial spasm. Embolism, perhaps from the site of femoral artery repair, is also unlikely. There was never any evidence of emboli in the legs of any of our patients.

Trauma to muscle, the commonly favoured causative factor, when the syndrome occurs after exercise was almost certainly not a major factor in our patients. Two possibilities remain:

DIRECT ISCHAEMIA In our patients this appears to be the most likely cause. The lesions all occurred on the limb being perfused, the femoral artery thereby being occluded. In cases 1 and 4 the profunda femoris artery and much of the collateral circulation to the limb was occluded. Possibly the gluteal collateral circulation would be at least partially occluded by the weight of the patient during the operation.

The patients all had relatively long bypass times. It is interesting that all patients were young and relatively active. Cases 1 and 4 had significant atheroma. The acute anterior tibial compartment syndrome following exercise is very uncommon in people over 50 years.

VENOUS STASIS This has been described as a factor in the aetiology of the syndrome. Recently
Kéry and Wouters (1971) suggested that identical pathological changes can occur from venous obstruction. It seems unlikely that pressure from the cannulated femoral artery could occlude the adjacent femoral vein, and it would be surprising if this was a significant factor as the timing and is distribution of the signs and symptoms are most $\vec{\circ}$ unlike those of venous stasis or thrombosis.

Although we have no conclusive evidence for the $\overrightarrow{\vec{\omega}}$ aetiology of the anterior tibial compartment syn- $\sigma$ drome in our patients, it seems probable that pro- $\overrightarrow{\vec{x}}$ longed arterial occlusion is the major initiating $\underset{\infty}{\sim}$ factor.

The results in our patients support the contention of Leach, Zohn, and Stryker (1964) that $N$ early surgical treatment is essential. Equally important when the femoral artery is selected as the perfusion site, it should be cannulated when- $\subseteq$ ever possible without occluding the profunda femoris in order to ensure adequate collateral $\overrightarrow{0}$ flow, particularly if a long bypass time is expected. $\omega$

We should like to thank Mr. O. S. Tubbs, F.R.C.S., and Mr. I. M. Hill, F.R.C.S., for permission to describe their cases.

\section{REFERENCES}

Carter, A. B., Richards, R. L., and Zachary, R. B. (1949). The anterior tibial syndrome. Lancet, 2, 928.

Kéry, L., and Wouters, H. W. (1971). Effect of venous stasis on striated muscles and its relation to the anterior tibial syndrome. Acta Chirurgica Academiae Scientiarurn Hungaricae, 12, 151.

Leach, R. E., Zohn, D. A., and Stryker, W. S. (1964). 의 Anterior tibial compartment syndrome. Archives of Surgery, 88, 187.

Vogt, P. R. (1943). Ischaemic muscular necrosis following marching. Read before the Oregon State Medicalo Society 4 September, 1943. 